\title{
Cytokine Expression of Microscopic Colitis Including Interleukin-17
}

\author{
Eunkyoung Park*, Young Sook Park*, Dae Rim Park*, Sung Ae Jung ${ }^{\dagger}$, Dong Soo Han , Byung Ik Jang ${ }^{\S}$, Young Ho Kim", \\ Won Ho Kim", Yun ju Jo*, Ki Ho Lee ${ }^{*}$, Won Mi Lee**, Eun Kyung Kim**, and Hae Soo Koo ${ }^{\text {tt }}$ \\ "Department of Internal Medicine, Eulji University School of Medicine, ${ }^{\dagger}$ Department of Internal Medicine, Ewha University School of Medicine, \\ ${ }^{\ddagger}$ Department of Internal Medicine, Hanyang University School of Medicine, Seoul, ${ }^{s}$ Department of Internal Medicine, Yeungnam University \\ School of Medicine, Daegu, "Department of Internal Medicine, Sungkyunkwan University School of Medicine, "Department of Internal \\ Medicine, Yonsei University School of Medicine, " Department of Biochemistry, Eulji University School of Medicine, ***Department of Pathology, \\ Eulji University School of Medicine, and ${ }^{t \dagger}$ Department of Pathology, Ewha Womans University School of Medicine, Seoul, Korea
}

Background/Aims: Microscopic colitis is characterized by chronic watery diarrhea with specific pathological changes that can be diagnosed by microscopic examination. We performed immunohistochemical analysis of proinflammatory cytokines to investigate the pathogenic mechanism of microscopic colitis. Methods: This study consisted of six patients with lymphocytic colitis, six patients with collagenous colitis, and six patients with functional diarrhea but normal pathology. We performed an immunohistochemical analysis of the colonic mucosal biopsies to assess the expression of cyclo-oxygenase-2, interleukin-17, nuclear factor- $\kappa \mathrm{B}$, interferon- $\gamma$, inducible nitric oxide synthase, and tumor necrosis factor- $\alpha$. We compared the quantity score of immunohistochemical staining among the groups. Results: The microscopic colitis group showed significantly higher expression of cyclo-oxygenase-2, interleukin-17, nuclear factor$\kappa \mathrm{B}$, and interferon $\gamma$ compared with the control group. Cytokine expression was similar between collagenous colitis and lymphocytic colitis. However, the expression of cyclo-oxygenase-2 was higher in collagenous colitis. Conclusions: Proinflammatory cytokines, including interleukin-17 and interferon- $\gamma$, are highly expressed in microscopic colitis. The expression of cyclo-oxygenase-2 was higher in collagenous colitis than in lymphocytic colitis. This study is the first on interleukin-17 expression in microscopic colitis patients. (Gut Liver 2015;9:381-387)

Key Words: Colitis, collagenous; Immunohistochemistry; Colitis, Iymphocytic; Colitis, microscopic

\section{INTRODUCTION}

Microscopic colitis (MC) is a chronic inflammatory bowel disease with unknown etiology characterized by chronic watery diarrhea without gross abnormalities on endoscopic examination. ${ }^{1}$ The histological classification of MC reveals two distinct diseases: collagenous colitis (CC) and lymphocytic colitis (LC). CC is defined by colonic intraepithelial lymphocytosis and increased numbers of inflammatory cells within the lamina propria, which develops a thickened subepithelial collagen band. Intraepithelial lymphocytosis is also evident in LC; however, there is no subepithelial collagen band. ${ }^{2}$

The pathogenesis of MC remains unknown. Moreover, it is not clear whether CC and LC are the same disease entity. Some evidence suggests that MC occurs as a response to a luminal antigen, such as bile acid, toxins, colonic infections, and medications, including nonsteroidal anti-inflammatory drugs and proton pump inhibitors. ${ }^{3}$ These causative factors increase luminal permeability and subsequent inflammatory responses in the lamina propria. ${ }^{4,5}$

The cytokine profile of MC has not been fully evaluated. A few studies have investigated the cytokine profile of MC, including $\mathrm{T}$ helper cell type 1 mucosal cytokine, interferon- $\gamma$ (IFN- $\gamma$ ), nuclear factor- $\kappa \mathrm{B}(\mathrm{NF}-\kappa \mathrm{B})$, cyclo-oxygenase-2 (COX-2), and nitric oxide synthases (NOS). These studies showed CC had high expression levels of NF- $\kappa \mathrm{B}$, iNOS, and COX-2.,6-8 However, most of these studies only assessed CC. Thus, we aimed to evaluate the expressions of various proinflammatory cytokines known to be associated with inflammatory bowel disease (IBD) in both CC and LC patients by immunohistochemical evaluation.

\footnotetext{
Correspondence to: Young Sook Park

Department of Internal Medicine, Eulji University School of Medicine, 68 Hangeulbiseok-ro, Nowon-gu, Seoul 139-711, Korea

Tel: +82-2-970-8207, Fax: +82-2-972-8621, E-mail: pys1109@eulji.ac.kr

Received on December 7, 2013. Revised on January 29, 2014. Accepted on March 3, 2014. Published online on July 25, 2014

pISSN 1976-2283 eISSN 2005-1212 http://dx.doi.org/10.5009/gnl13439

(a) This is an Open Access article distributed under the terms of the Creative Commons Attribution Non-Commercial License (http://creativecommons.org/licenses/by-nc/3.0) which permits unrestricted non-commercial use, distribution, and reproduction in any medium, provided the original work is properly cited.
} 


\section{MATERIALS AND METHODS}

\section{Study populations/tissue specimens}

All patients presented with chronic watery diarrhea for more than 4 weeks. Colonoscopy showed that all mucosa were normal or nearly normal. A colonic mucosal biopsy was done randomly at a point between the ascending colon and the rectum, and the samples were immediately embedded in formalin. The LC and CC groups were each comprised of 6 patients with histological evidence for their respective diagnosis. The control group consisted of six patients with functional diarrhea neither any histological evidence of MC nor irritable bowel syndrome with diarrhea by Rome III criteria.

\section{Diagnostic criteria of MC}

LC was diagnosed on the basis of the histological findings of colonic mucosal biopsy specimens, including $>20$ intraepithelial lymphocytic infiltrations per 100 epithelial cells, inflammation in the lamina propria consisting of lymphocytes and plasma cells, and an absent subepithelial collagen layer or $<10-\mu \mathrm{m}$ subepithelial collagen layer. CC was diagnosed if the colonic mucosal biopsy specimen revealed a subepithelial collagen layer $>10 \mu \mathrm{m} .^{9-11}$ All biopsy specimens were evaluated by the same pathologist.

\section{Immunohistochemistry}

Sections of formalin-fixed, paraffin-embedded tissue samples (4 to $5 \mu \mathrm{m}$ ) were dewaxed in xylene 3 times and thoroughly hydrated through a series of 100\% ethanol twice, 70\% ethanol once, and distilled $\mathrm{H}_{2} \mathrm{O}\left(\mathrm{dH}_{2} \mathrm{O}\right)$ once. Sections were then subjected to an antigen-retrieval step that consisted of a 10-minute microwave oven treatment in citrate buffer. After sections were cooled at room temperature, they were washed in phosphatebuffered saline (PBS). Next, endogenous peroxidases were inactivated in 3\% hydrogen peroxide $\left(\mathrm{H}_{2} \mathrm{O}_{2}\right)$ /methanol solutions, and the sections were washed in PBS 3 times. Sections were blocked in goat or rabbit serum and incubated with primary antibodies (all diluted 1:100; Santa Cruz Biotechnology, Santa Cruz, CA, USA) against COX-2, tumor necrosis factor- $\alpha$ (TNF- $\alpha$ ), interleukin-17 (IL-17), inducible nitric oxide synthase (iNOS), NF- $\mathrm{B}$, or IFN- $\gamma$ overnight at $4^{\circ} \mathrm{C}$. After removing the primary antibody by washing in PBS, the sections were incubated with an appropriate biotin-conjugated secondary antibody (1:100) for 1 hour at room temperature. Next, the secondary antibody was removed by washing in PBS, and the avidin-biotin complex was applied for 30 minutes at room temperature. Diaminobenzidine was used to develop the color. After stopping the color reaction in $\mathrm{dH}_{2} \mathrm{O}$, slides were incubated with hematoxylin for counterstaining. The sections were then dehydrated in a series of 70\%, $80 \%, 90 \%$, and 100\% ethanol and cleared in xylene before they were mounted on microscope slides and cover-slipped. Images were acquired with a light microscope equipped with a digital camera.

\section{Immunohistochemical quantification}

There is no standardized method to evaluate immunohistochemical quantification, although previous studies have defined their quantification procedures. ${ }^{1,8}$ In this study, positive cells were counted to quantify the immunohistochemical results. Locations were classified on the basis of whether the positive cells were epithelial or inflammatory, and staining ratios were quantitatively assessed on a scale from 0 to 2 : 0 , negative, i.e., $<10 \%$ positive cells; 1 , weakly positive, between $10 \%$ and 50\% positive cells; and 2 , strongly positive, $>50 \%$ positive cells. After scoring each specimen, the total score was calculated as the sum of epithelial and inflammatory cell ratings.

\section{Statistical analysis}

SPSS version 22.0 (IBM Corp., Armonk, NY, USA) was used for all statistical analysis. Data are expressed as mean \pm standard deviation. Comparisons between two groups were performed with Mann-Whitney U tests. A p-value $<0.05$ was considered statistically significant.

\section{RESULTS}

Immunohistochemical stain of COX-2, IL-17, NF- $\kappa$ B, IFN- $\gamma$, iNOS, and TNF- $\alpha$ was performed using colonoscopic biopsy specimen of MC and control with quantification scoring of staining results (Table 1).

COX-2, IL-17, IFN- $\gamma$, iNOS, and TNF- $\alpha$ were stained at cytoplasm of cells. NF- $\kappa B$ shows nuclear staining.

The expression of proinflammatory cytokines, including IL-17 and IFN- $\gamma$ were significantly increased in MC. The expression of COX -2 and NF- $-\mathrm{B}$ also increased significantly in MC. iNOS was expressed weakly on CC. TNF- $\alpha$ was not well expressed both

Table 1. Comparison of the Quantification of Immunochemical Staining among the Groups

\begin{tabular}{lccccc}
\hline & \multirow{2}{*}{ Control } & \multicolumn{3}{c}{ MC } & \\
\cline { 3 - 5 } & & LC & CC & p-value & p-value \\
& & & & \\
\hline COX-2 & $1.67 \pm 0.52$ & $2.00 \pm 1.79$ & $4.00 \pm 0.00$ & 0.045 & 0.022 \\
IL-17 & $0.70 \pm 0.00$ & $1.67 \pm 0.52$ & $1.67 \pm 1.03$ & 0.001 & 0.727 \\
NF- $\mathrm{NB}$ & $0.00 \pm 0.00$ & $1.00 \pm 0.00$ & $1.00 \pm 0.00$ & 0.001 & 1.000 \\
IFN- $\gamma$ & $1.33 \pm 1.03$ & $3.33 \pm 0.52$ & $3.00 \pm 0.89$ & 0.002 & 0.484 \\
iNOS & $0.00 \pm 0.00$ & $0.00 \pm 0.00$ & $0.67 \pm 0.52$ & 0.119 & 0.019 \\
TNF- $\alpha$ & $0.00 \pm 0.00$ & $0.00 \pm 0.00$ & $0.00 \pm 0.00$ & 1.000 & 1.000 \\
\hline
\end{tabular}

MC, microscopic colitis; LC, lymphocytic colitis; CC, collagenous colitis; COX-2, cyclo-oxygenase-2; IL-17, interleukin-17; NF- $\kappa \mathrm{B}$, nuclear factor- $\kappa \mathrm{B}$; INF- $\gamma$, interferon- $\gamma$; iNOS, inducible nitric oxide synthase; TNF- $\alpha$, tumor necrosis factor- $\alpha$.

${ }^{*}$ Comparison between control and MC groups; ${ }^{\dagger}$ Comparison between LC and CC groups. 

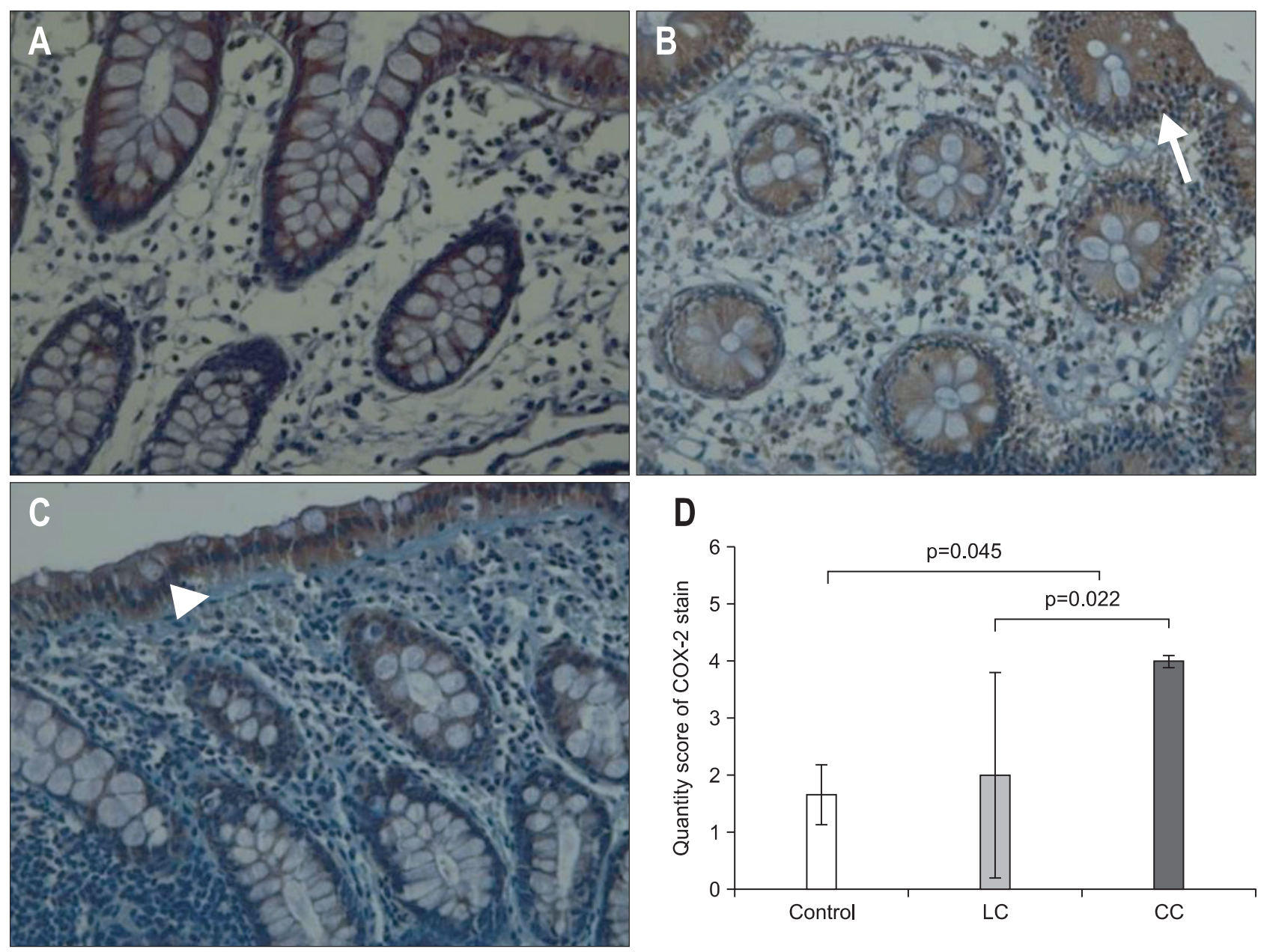

Fig. 1. Immunohistochemical staining results for cyclo-oxygenase-2 (COX-2) ( $\times 400)$. Immunohistochemical analysis comparing the levels of COX-2 in control (A), lymphocytic colitis (LC) (B), and collagenous colitis (CC) (C). The positive expression of COX-2 was brown granules deposited in the cytoplasm of the epithelial and inflammatory cells. The arrow indicates intraepithelial lymphocytosis in LC (B). The arrowhead shows the subepithelial collagen layer in CC (C). The graph shows the average quantity score of each group (D). The microscopic colitis group showed an increased quantity score compared to the control group ( $\mathrm{p}=0.045)$. The CC group showed an increased quantity score compared to the LC group $(\mathrm{p}=0.022)(\mathrm{D})$.

MC and control groups.

For COX-2, the immunohistochemical analysis yielded an epithelial cell quantity score of 1 and an inflammatory cell quantity score of 0 , for a total score of 1 in the control group (Fig. 1A). In the LC group, the epithelial cell score was 1, the inflammatory cell score was 1 , and the total score was 2 (Fig. 1B). The epithelial and inflammatory cell scores were 2 and 2, respectively, for a total score of 4 in the CC group (Fig. 1C).

Compared to the control group, there was significantly higher expression in CC and LC ( $\mathrm{p}=0.045)$ (Fig. 1D). All other cytokines were quantified using the same protocol.

For IL-17, the epithelial and inflammatory cell scores were 1 and 0 in the control group, respectively (Fig. 2A). In the LC group, both the epithelial and inflammatory cell scores were 1, for a total score of 2 (Fig. 2B). In the CC group, the epithelial cell score was 2, and the inflammatory cell score was 1 , for total score of 3 (Fig. 2C). There was a statistically significant differ- ence between control and MC groups ( $\mathrm{p}=0.001$ ) (Fig. 2D).

For $N F-\kappa B$, the total score was 0 in the control group (Fig. $3 \mathrm{~A}$ ), and expression was increased in the MC group. In the LC and CC group, only the epithelial cell count was increased, and the total score was 1 (Fig. 3B and C). The MC group showed increased staining intensity compared the control group $(p=0.001)$ (Fig. 3D).

Comparing between CC and LC, expression of IL-17, IFN- $\gamma$, and TNF- $\alpha$ was not significantly different. However, CC patients showed higher expression levels of COX-2 and iNOS than LC patients (Table 1) $(\mathrm{p}<0.05)$.

\section{DISCUSSION}

MC has two main entities: CC and LC. Microscopically, LC is characterized by a marked intraepithelial lymphocytosis, whereas CC exhibits increased subepithelial collagen deposition. 

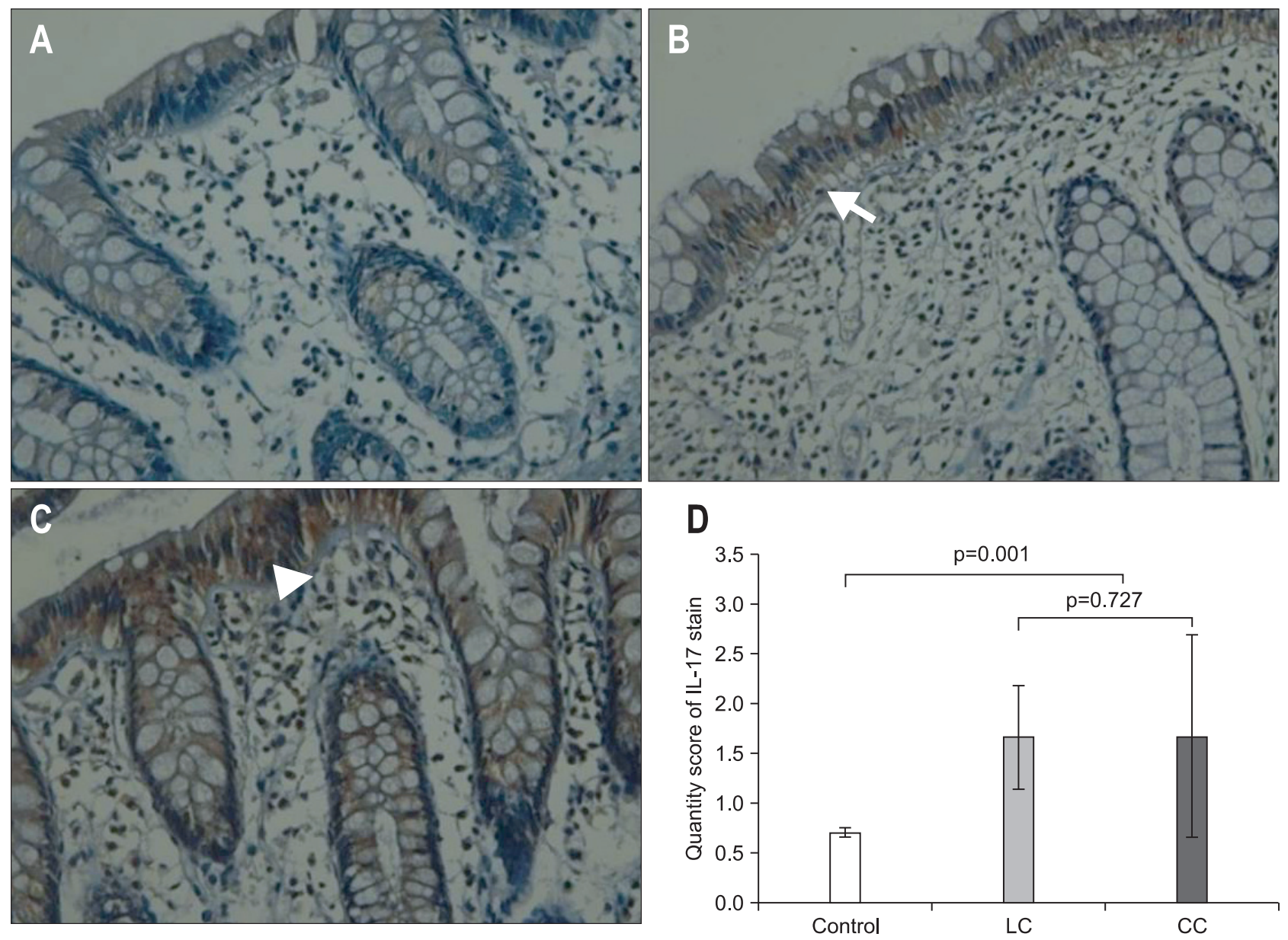

Fig. 2. Immunohistochemical staining results for interleukin-17 (IL-17) $(\times 400)$. Immunohistochemical analysis comparing levels of IL-17 in control (A), lymphocytic colitis (LC) (B), and collagenous colitis (CC) (C). The positive expression of IL-17 is visualized as brown granules deposited in the cytoplasm of the epithelial and inflammatory cells. The arrow indicates intraepithelial lymphocytosis in LC (B). The arrowhead shows the subepithelial collagen layer in CC (C). The graph shows the average quantity score of each group (D). The microscopic colitis group showed an increased quantity score compared with the control group ( $\mathrm{p}=0.001)$. There were no significant differences between the LC and CC groups ( $\mathrm{p}=0.727)$ (D).

The pathogenesis of MC is not fully understood. Although the cytokine profile of IBD has been well studied, little is known about the inflammatory mediators involved in MC pathogenesis as relatively few studies have been performed. One report stated that MC patients predominantly had a Th1 type cytokine profile with marked increases in IFN- $\gamma$, IL 15, TNF- $\alpha$, and iNOS. ${ }^{12}$ Other studies focused on abnormal immunomodulation in MC patients, including the evaluation of proinflammatory cytokines. Specifically, CC exhibited increased activities of NF- $\kappa \mathrm{B}$, iNOS, IFN- $\gamma$, and COX-2. Also, LC demonstrated increased IFN- $\gamma \cdot{ }^{1,6-8}$

To our knowledge, there are few reports comparing cytokine profiles between the two MC disease entities. We performed immunohistochemical studies to compare proinflammatory cytokine expression levels between CC and LC. Our analysis revealed that the expression levels of COX-2, IL-17, NF- $\kappa \mathrm{B}$, and IFN- $\gamma$ were increased in the MC group compared with the control group. Also, COX-2 and iNOS expression levels were higher in CC than LC.

COX-2, a prostaglandin synthase enzyme, is normally found at very low levels but is rapidly induced by a number of stimuli, including cytokines, growth factors, hormones, and carcinogens and is believed to be responsible for the production of prostaglandins that mediate inflammation. ${ }^{8,13}$ A previous study described a clear relationship between IBD activity and COX-2 expression. ${ }^{14}$ COX-2 predominates under inflammatory conditions and is also induced in cancer cells. ${ }^{15}$ Our study also showed COX-2 expression was strong in the MC groups and this was especially prominent for CC. This finding suggests that COX-2 and prostaglandin synthesis might be involved in MC pathogenesis. One previous study assessed CC samples and determined that only inflammatory cells in the lamina propria were COX-2 positive. $^{8}$ Others have shown that COX-2 expression is also increased in epithelial cells in ulcerative colitis and Crohn's disease. ${ }^{16,17}$ In our study, COX-2 expression levels were increased both in epithelial and inflammatory cells in the LC and CC groups, especially COX-2 expression in CC was significantly stronger than LC ( $\mathrm{p}=0.022)$.

This is the first study of IL-17 expression in MC as far as we 

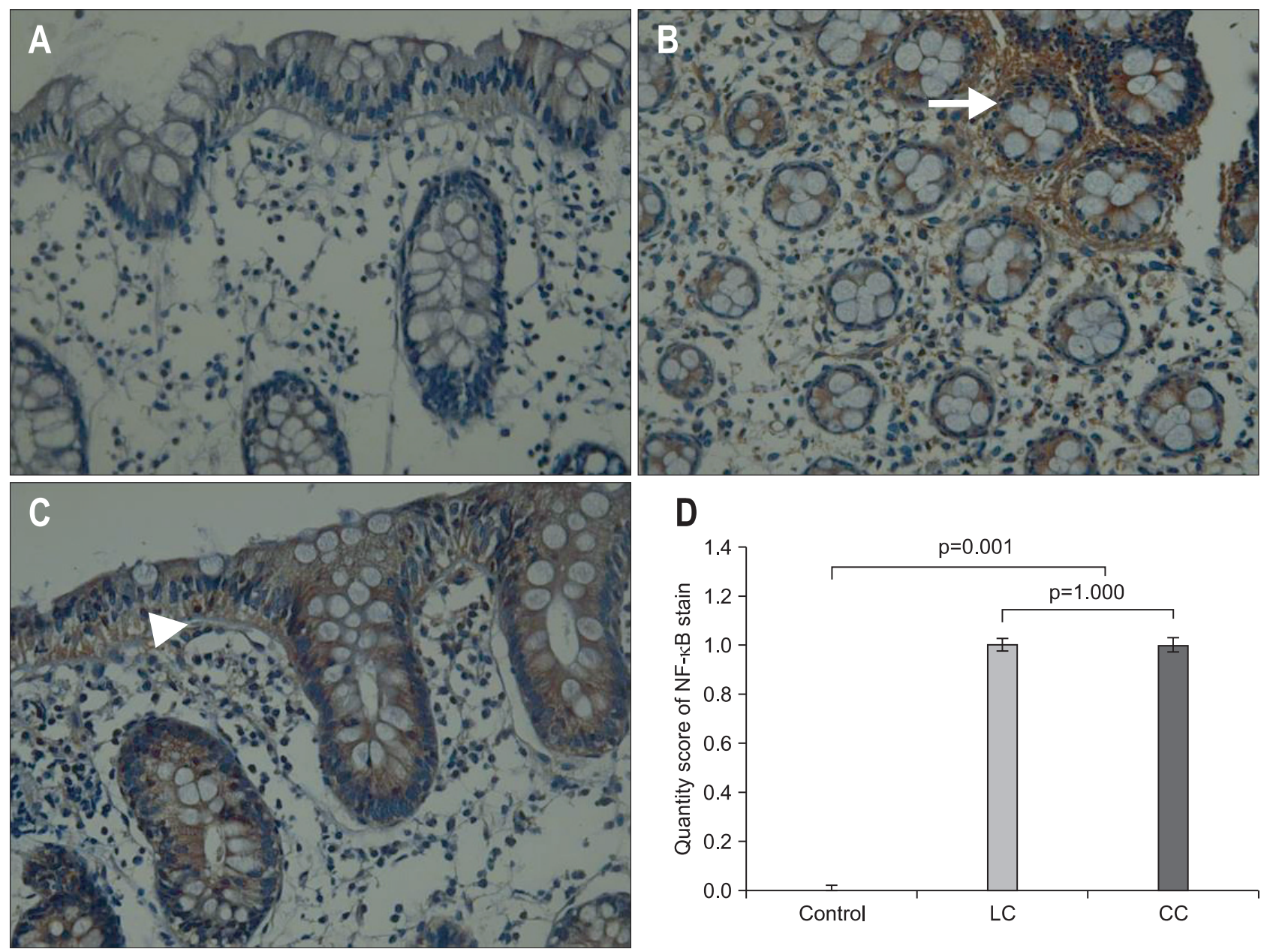

Fig. 3. Immunohistochemical staining results for nuclear factor- $\kappa B(N F-\kappa B)(A, x 400 ; B, \times 200 ; C, \times 400)$. Immunohistochemical analysis comparing the levels of NF- $\kappa$ B in control (A), lymphocytic colitis (LC) (B), and collagenous colitis (CC) (C). The positive expression of NF- $\kappa$ B is visualized as brown granules deposited in the nucleus of epithelial cells. The arrow indicates intraepithelial lymphocytosis in LC (B). The arrowhead shows the subepithelial collagen layer in CC (C).The graph shows the average quantity score of each group (D). The microscopic colitis group showed an increased quantity score compared with the control group $(\mathrm{p}=0.001)$. There were no significant differences between the LC and CC groups ( $p=1.000)$ (D).

know. IL-17, which is produced by Th17 cells, serves an important function in antimicrobial immunity at mucosal barriers by stimulating production of antimicrobial proteins by epithelial cells and neutrophil recruitment. However, because of its inflammatory nature, it is highly pathogenic in excess amounts and has been demonstrated to be a major player in IBD pathogenesis. ${ }^{18}$ Also, an elevated level of IL-17 was found in patients with IBD. ${ }^{19} \mathrm{IL}-17$ plays a potential role in the amplification of intestinal inflammation by stimulating endothelial cells, myofibroblasts, and epithelial cells. ${ }^{20,21}$ It also has important effects on intestinal permeability. One study reported that the mRNA level of IL-17 was increased in MC patients. ${ }^{18}$ In our study, we observed that IL-17 expression was increased in MC, both in epithelial cells and inflammatory cells, this result suggests that IL-17 also has an important role in MC pathogenesis, similar to IBD. Recently, an attempt to therapeutically inhibit IL-17 production was performed in IBD patients. Vidofludimus, a novel small molecule inhibitor of dihydroorotate dehydrogenase, inhibited IL-17 production in activated lymphocytes and attenuated various parameters of acute TNBS (2,4,6-trinitrobenzenesulfonic acid)-induced colitis in mice. ${ }^{22}$ Our study shows that IL17 regulation is another viable therapeutic option to treat MC.

$\mathrm{NF}-\kappa \mathrm{B}$ is a major transcription factor. NF- $\kappa \mathrm{B}$ translocates into the nucleus where it binds to the promoter region of multiple genes with predominantly proinflammatory actions. In our study, the expression of $\mathrm{NF}-\kappa \mathrm{B}$ was significantly increased in both CC and LC. It suggested that proinflammatory cytokines was important for pathogenesis of MC. ${ }^{1}$

IFN- $\gamma$ has a critical effect on intestinal permeability. In previous study, MC was likely to have a T helper cell type 1 cytokine profile including a predominance of IFN- $\gamma$, a lesser increase in TNF- $\alpha$ and increases in IL-15, iNOS and possibly IL-10. In our study, the expression of IFN- $\gamma$ showed significantly increase in MC similar with that result. ${ }^{6}$ 
TNF- $\alpha$ expression in MC has only been investigated in two studies, both of which showed that it was increased in MC. ${ }^{12,18}$ But, in our study TNF- $\alpha$ expression was very weak, less than $10 \%$ stained cell in both epithelial and inflammatory cells in only CC. In LC, only one specimen expressed TNF- $\alpha$ on inflammatory cells in MC. TNF- $\alpha$ is the most well-known proinflammatory mediator involved in the pathogenesis of IBD. It is released by activated macrophages and $\mathrm{T}$ cells and triggers a chain of cellular mediators that cause intestinal damage. ${ }^{23}$ TNF- $\alpha$ has been reported to promote inflammation. ${ }^{24}$ For this reason, anti-TNF- $\alpha$ has been used in the last decade to treat IBD that is refractory to conventional treatment. ${ }^{25}$ But, according to our study, the role of anti-TNF therapies in MC may be less valuable than IBD. Even though a few case reports have shown that anti-TNF therapies were effective in severe refractory $\mathrm{MC} .^{26,27}$ Further study will be necessary to confirm the role of anti-TNF- $\alpha$ in MC.

In this study, we verified the increased expression of proinflammatory cytokines such as IL-17, and IFN- $\gamma$ in MC patients, suggesting that MC and IBD shares similar proinflammatory cytokine profiles. These proinflammatory cytokines are known to be important in the pathogenesis of IBD patients. Because IL-17 has been a key role in IBD, result of this study lead new insight of understanding pathogenesis of MC.

Our study has some limitations. One is that we used control group with functional diarrhea. Another is we could not confirm quantitative cytokine expression such as real-time polymerase chain reaction technique. Also we could not compare the cytokine expression with clinical disease activity due to limited clinical information.

In conclusion, MC showed increased expression of proinflammatory cytokines including COX-2, IL-17, NF- $\kappa$ B, and IFN- $\gamma$ compared to controls, which is similar with IBD. But contrary to IBD, TNF- $\alpha$ is not significantly expressed in both CC and LC. Also cytokine profiles between CC and LC didn't show any significant difference. COX-2 and iNOS showed relatively higher expression in CC than LC in immunohistochemical stain which will be confirmed by further study.

\section{CONFLICTS OF INTEREST}

No potential conflict of interest relevant to this article was reported.

\section{ACKNOWLEDGEMENTS}

This study was supported by 2009 Eulji Research Grant (EJRG-09-014-12E11).

\section{REFERENCES}

1. Andresen L, Jørgensen VL, Perner A, Hansen A, Eugen-Olsen J,
Rask-Madsen J. Activation of nuclear factor kappaB in colonic mucosa from patients with collagenous and ulcerative colitis. Gut 2005;54:503-509.

2. Kao KT, Pedraza BA, McClune AC, et al. Microscopic colitis: a large retrospective analysis from a health maintenance organization experience. World J Gastroenterol 2009;15:3122-3127.

3. Loftus EV. Microscopic colitis: epidemiology and treatment. Am J Gastroenterol 2003;98(12 Suppl):S31-S36.

4. Thomson RD, Lestina LS, Bensen SP, Toor A, Maheshwari Y, Ratcliffe NR. Lansoprazole-associated microscopic colitis: a case series. Am J Gastroenterol 2002;97:2908-2913.

5. Yagi K, Nakamura A, Sekine A, Watanabe H. Nonsteroidal antiinflammatory drug-associated colitis with a histology of collagenous colitis. Endoscopy 2001;33:629-632.

6. Tagkalidis PP, Gibson PR, Bhathal PS. Microscopic colitis demonstrates a T helper cell type 1 mucosal cytokine profile. J Clin Pathol 2007;60:382-387.

7. Perner A, Andresen L, Normark M, et al. Expression of nitric oxide synthases and effects of L-arginine and L-NMMA on nitric oxide production and fluid transport in collagenous colitis. Gut 2001;49:387-394.

8. Wildt S, Rumessen JJ, Csillag C, Normark M, Poulsen KA, Kolko M. Cyclooxygenase-2 immunoreactivity in collagenous colitis. APMIS 2009;117:500-506.

9. Lazenby AJ, Yardley JH, Giardiello FM, Jessurun J, Bayless TM. Lymphocytic ("microscopic") colitis: a comparative histopathologic study with particular reference to collagenous colitis. Hum Pathol 1989;20:18-28.

10. Giardiello FM, Lazenby AJ. The atypical colitides. Gastroenterol Clin North Am 1999;28:479-490, x.

11. Jessurun J, Yardley JH, Giardiello FM, Hamilton SR, Bayless TM. Chronic colitis with thickening of the subepithelial collagen layer (collagenous colitis): histopathologic findings in 15 patients. Hum Pathol 1987;18:839-848.

12. Dey I, Beck PL, Chadee K. Lymphocytic colitis is associated with increased pro-inflammatory cytokine profile and up regulation of prostaglandin receptor EP4. PLoS One 2013;8:e61891.

13. Wallace JL. Prostaglandin biology in inflammatory bowel disease. Gastroenterol Clin North Am 2001;30:971-980.

14. Hendel J, Nielsen OH. Expression of cyclooxygenase-2 mRNA in active inflammatory bowel disease. Am J Gastroenterol 1997;92: 1170-1173.

15. Müller-Decker K, Albert C, Lukanov T, Winde G, Marks F, Fürstenberger G. Cellular localization of cyclo-oxygenase isozymes in Crohn's disease and colorectal cancer. Int J Colorectal Dis 1999;14:212-218.

16. Singer II, Kawka DW, Schloemann S, Tessner T, Riehl T, Stenson WF. Cyclooxygenase 2 is induced in colonic epithelial cells in inflammatory bowel disease. Gastroenterology 1998;115:297-306.

17. Roberts PJ, Morgan K, Miller R, Hunter JO, Middleton SJ. Neuronal COX-2 expression in human myenteric plexus in active inflammatory bowel disease. Gut 2001;48:468-472. 
18. Kumawat AK, Strid H, Tysk C, Bohr J, Hörnquist EH. Microscopic colitis patients demonstrate a mixed Th17/Tc17 and Th1/Tc1 mucosal cytokine profile. Mol Immunol 2013;55:355-364.

19. Kim SW, Kim ES, Moon CM, et al. Genetic polymorphisms of IL23R and IL-17A and novel insights into their associations with inflammatory bowel disease. Gut 2011;60:1527-1536.

20. Zhang Z, Zheng M, Bindas J, Schwarzenberger P, Kolls JK. Critical role of IL-17 receptor signaling in acute TNBS-induced colitis. Inflamm Bowel Dis 2006;12:382-388.

21. Strzępa A, Szczepanik M. IL-17-expressing cells as a potential therapeutic target for treatment of immunological disorders. Pharmacol Rep 2011;63:30-44.

22. Fitzpatrick LR. Novel pharmacological approaches for inflammatory bowel disease: targeting key intracellular pathways and the IL-23/IL-17 axis. Int J Inflam 2012;2012:389404.
23. Casellas i Jordà F. TNF-alpha inhibitors in inflammatory bowel disease. Med Clin (Barc) 2004;123:627-634.

24. Ullman TA, Itzkowitz SH. Intestinal inflammation and cancer. Gastroenterology 2011;140:1807-1816.

25. Malik T, Mannon P. Inflammatory bowel diseases: emerging therapies and promising molecular targets. Front Biosci (Schol Ed) 2012;4:1172-1189.

26. Esteve M, Mahadevan U, Sainz E, Rodriguez E, Salas A, Fernández-Bañares F. Efficacy of anti-TNF therapies in refractory severe microscopic colitis. J Crohns Colitis 2011;5:612-618.

27. Aram G, Bayless TM, Chen ZM, Montgomery EA, Donowitz M, Giardiello FM. Refractory lymphocytic enterocolitis and tumor necrosis factor antagonist therapy. Clin Gastroenterol Hepatol 2010;8:391-394. 